\title{
A REMARK ON AN INEQUALITY FOR THE PRIME COUNTING FUNCTION
}

\author{
DIETRICH BURDE
}

Abstract. We note that the inequalities $0.92 \frac{x}{\log (x)}<\pi(x)<1.11 \frac{x}{\log (x)}$ do not hold for all $x \geqslant 30$, contrary to some references. These estimates on $\pi(x)$ came up recently in papers on algebraic number theory. conjecture.

Mathematics subject classification (2000): Chebyshev-type estimates on primes, Serre's modularity Key words and phrases: 11N05, 11A41.

\section{REFERENCES}

[1] T. APOSTOL, Introduction to analytic number theory, Springer Verlag, fünfte Auflage (1998).

[2] P. L. ChebYshev, Mémoire sur les nombres premiers, Journal de Math. Pures et Appl., 17, (1852), 366-390.

[3] K. ChANDRASEKHARAN, Introduction to analytic number theory, Moscow, (1974).

[4] P. DusART, Inégalités explicites pour $\psi(X), \theta(X), \pi(X)$ et les nombres premiers, C. R. Math. Acad. Sci. Soc. R. Can., 21, (2) (1999), 53-59.

[5] P. DUSART, Autour de la fonction qui compte le nombre de nombres premiers, Thése de Doctorat de l’Université de Limoges (1998).

[6] W. ElLison, F. Ellison, Prime numbers, Wiley Interscience, (1985).

[7] M. HassanI, Approximation of $\pi(x)$ by $\Psi(x)$, J. of Inequ. in Pure and Apl. Math., 7, (1) (2006), 1-7.

[8] C. KHARE, On Serre's modularity conjecture for 2 -dimensional mod p representations of $G a l(\overline{\mathbb{Q}} / \mathbb{Q})$ unramified outside $p$, ArXiv math.NT/0504080 (2005).

[9] C. KHARE, Serre's modularity conjecture: the level one case, To appear in Duke Math. J.

[10] L. PANAitopol, Several approximations of $\pi(x)$, Math. Inequal. Appl., 2, (3) (1999), 317-324.

[11] K. PRACHAR, Primzahlverteilung, Grundlehren der Mathematischen Wissenschaften 91, Springer-Verlag, Berlin-New York, (1978).

[12] J. B. Rosser, L. SCHOENFELD, Sharper bounds for the Chebyshev functions $\theta(x)$ and $\psi(x)$, Math. Comp., (29) (1975), 243-269.

[13] J. SÁNDOR, D. S. MitRinOVIC AND B. CRSTICI, Handbook of number theory I, Springer, (2006).

[14] G. TENENBAUM, Introduction to analytic and probabilistic number theory, Cambridge studies in advanced mathematics, 46, (1995). 